\title{
On-line Analysis for Agricultural Production Image Data
}

\author{
Ronghua $\mathrm{GaO}^{1,2,3,4, \text { a }}$, Huarui $\mathrm{Wu}^{1,2,3,4, \mathrm{~b}^{*}}$ \\ ${ }^{1}$ Beijing Research Center for Information Technology in Agriculture, Beijing Academy of Agriculture \\ and Forestry Sciences, Beijing, 100097, China \\ ${ }^{2}$ National Engineering Research Center for Information Technology in Agriculture, Beijing 100097, \\ China \\ ${ }^{3}$ Key Laboratory of Agri-informatics, Ministry of Agriculture, Beijing 100097, China \\ ${ }^{4}$ Beijing Engineering Research Center of Agricultural Internet on Things, Beijing 100097, China \\ agaorh@nercita.org.cn, ${ }^{b}$ wuhr@nercita.org.cn(*corresponding author)
}

Keywords: image segmentation; frequency domain; entropy; image characteristic; Fourier transformation

\begin{abstract}
Image plays an important role in agricultural production data. It is a good guidance that some valuable knowledge from that images by online analysis. As a crucial procedure in which images are divided into distinct non-overlapping regions and the interested objects are extracted in the process of image analyzing, image recognizing and image segmentation. Automatic object segmentation is a hard work, for it is difficult for a computer to determine which is objective and which is not automatic. In this paper, the method of on-line analysis for agricultural production image data is proposed, and as an example image online segmentation based on frequency domain. An image transformation can be applied to an image to convert it from one domain to another. Viewing an image in domains such as frequency or Hough space enables the identification of features that may not be as easily detected in the spatial domain. Experimental results show that this method cannot only obtain a good result with general images, but also greatly reduce the computation cost for a large size image.
\end{abstract}

\section{Introduction}

Object extraction via image segmentation plays a key role in image online analysis. However, it remains a difficult problem. Target applications vary in the necessary degrees of precision, efficiency, and feature information required from image segmentation, so different algorithms are typically better suited to different applications. Image segmentation divides the images into their own characteristics' regions, and extracts the interested target. During the intelligent diagnosis of crop disease, it is important that effective segmentation of a complex background and the result will affect the extraction and disease identification.

Image segmentation is an important task in computer vision, pattern matching, and video coding[1]. The performance of computer vision is significantly influenced by the result of image segmentation.

Traditionally, the image is partitioned into several components in the way of minimizing some specific cost function of the vertices in the components or the boundary between the components. The common way of partitioning an image is to recursively partition the image into more than two components until the termination criterion is satisfied, that is known as bi-partitioning.

There are many research works in the area of image segmentation. The most relevant works include edge point grouping[2], region segmentation and merging[3] approaches that are susceptible to illumination variation, shadowing or changing of viewpoints, multivariate probabilistic modelling[4] that works well for simple transformation, and grouping method[5]. Many automatic image segmentation algorithms [6-8] have been proposed in the past as well. The common feature of these image segmentation methods is that they all make hypotheses about the image and test features, and make decisions by applying the thresholds explicitly or implicitly. For content-based image 
retrieval, segmentation schemes can be used to extract objects and their features from images, and then use the object feature information to increase image retrieval efficiency. A number of segmentation algorithms for building hierarchical image representations have been proposed. Although these hierarchical segmentation algorithms perform well in some specific areas, all of them suffer from various drawbacks. Nacken [9] and Shen et al. [10] employ methods similar to ours of iteratively grouping nodes in a hierarchical structure. However, their methods can lead to excessive iterations, generating a large number of hierarchy levels. Montanvert et al.[11] use stochastic analyses for image segmentation, but their segmentation results are unique due to the stochastic decimation applied at each level. Furthermore, all of the above algorithms use only the original pixel values when performing pixel clustering, which Marr argues is not sufficient [12].

In this paper, image segmentation based on frequency domain entropy is proposed. An image transformation can be applied to an image to convert it from one domain to another. Viewing an image in domains such as frequency or Hough space enables the identification of features that may not be as easily detected in the spatial domain. This method cannot only obtain a good result with general images, but also greatly reduce the computation cost for a large size image. In the future study, the properties and texture information of pixels will be used to further improve the segmented performance.

\section{Spatial Transformations of Image}

Spatial transformations are important in many aspects of functional image analysis. In functional imaging, particularly for functional magnetic resonance imaging, the signal changes due to any hemodynamic response can be small compared to signal changes that can result from subject motion, so prior to performing the statistical tests, it is important that the images are as closely aligned as possible.

Image Spatial Transform. Image space transformation for color image is to transform the image that is described by R-G-B form to a certain system. Image transformations are operations similar in concept to those for image enhancement. However, unlike image enhancement operations which are normally applied only to a single channel of data at a time, image transformations usually involve combining processing of data from multiple spectral bands. Arithmetic operations are performed to combine and transform the original bands into a new image which better display or highlight certain features of the scene.

An image transformation can be applied to an image to convert it from one domain to another. Viewing an image in domains such as frequency or Hough space enables the identification of features that may not be as easily detected in the spatial domain. Common image transforms include Hough Transform that is used to find lines in an image, Radon Transform that is used to reconstruct images from fan-beam and parallel-beam projection data, Discrete Cosine Transform that is used in image and video compression, Discrete Fourier Transform that is used in filtering and frequency analysis, and Wavelet Transform that is used to perform discrete wavelet analysis, denoise, and fuse images.

Fourier Transform. The Fourier transform, is a mathematical transformation employed to transform signals between spatial and frequency domain, which has many applications in physics and engineering. It is reversible, being able to transform from either domain to the other. The term itself refers to both the transform operation and the function it produces.

The Fourier Transform is an important image processing tool which is used to decompose an image into its sine and cosine components. The output of the transformation represents the image in the Fourier or frequency domain, while the input image is the spatial domain equivalent. In the Fourier domain image, each point represents a particular frequency contained in the spatial domain image.

The Fourier Transform is used in a wide range of applications, such as image analysis, image filtering, image reconstruction and image compression. 
In the case of a periodic function over time, the Fourier transform can be simplified to the calculation of a discrete set of complex amplitudes, called Fourier series coefficients. They represent the frequency spectrum of the original time-domain signal. Also, when a time domain function is sampled to facilitate storage or computer-processing, it is still possible to recreate a version of the original Fourier transform according to the Poisson summation formula, also known as discrete time Fourier transform. See also Fourier analysis and List of Fourier related transforms.

The Fourier transform has the following basic properties.

- Linearity, for any complex numbers $a$ and $b$,if $h(x)=a f(x)+b g(x)$,

then $h(\xi)=a f(\xi)+b g(\xi)$, where $h(\xi), f(\xi), g(\xi)$ is Fourier transforms of different functions respectively.

- Translation, for any real number $\varphi$,

if $h(x)=e^{i 2 \pi \times \varphi} f(x)$, then $h(\xi)=f(\xi-\varphi)$.

- Scaling, for non-zero real number $\kappa$, if $h(x)=f(\kappa x)$, then $h(\xi)=\frac{1}{|\kappa|} f\left(\frac{\xi}{\kappa}\right)$. The case $\kappa=-1$ lead to the time reversal property, which states if $h(x)=f(-x)$, then $h(\xi)=f(-\xi)$

- Conjugation, if $h(x)=\overline{f(x)}$, then $h(\xi)=\overline{f(-\xi)}$, in particular, if $f$ is real, then one has the reality condition $f(-\xi)=f(\xi)$, that is $f$ is a Hermitian function, and if $f$ is purely imaginary, then $f(-\xi)=-\overline{f(\xi)}$.

- Integration, substituting $\xi=0$ in the definition, we obtain $f(0)=\int_{-\infty}^{\infty} f(x) d x$, that is the evaluation of the Fourier transform in the origin equals the integral of $f$ all over its domain.

The Fourier Transform is used if we want to access the geometric characteristics of a spatial domain image. Because the image in the Fourier domain is decomposed into its sinusoidal components, it is easy to examine or process certain frequencies of the image, thus influencing the geometric structure in the spatial domain.

\section{Segmentation Based on Frequency Domain}

Image having pixel correlation is translated from image space to frequency space in this paper, defines rectangular grid $S=\{(m, n): 1 \leq m \leq M, 1 \leq n \leq N\}$ of a dimensional array $\{f(m, n):(m, n) \in S\}$ is of gray leaf image $I$ with the size $M \times N$, which $(m, n) \in S$ is a pixel coordinate on the grid, and $f(m, n)$ is a pixel gray level. Discrete Fourier transform of the image $I$ is as following.

$$
F(u, v)=\sum_{m=0}^{M-1} \sum_{n=0}^{N-1} f(m, n) e^{-j(2 \pi / N)(m u+n u)}
$$

Where $1 \leq u \leq M, 1 \leq v \leq N, j=\sqrt{-1}$, and spatial frequency energy (u,v) is defined as following.

$$
E_{u, v}=|F(u, v)|^{2}
$$

At this time, the frequency domain energy distribution of entropy which reflects the spatial frequency components in the frequency domain as following.

$$
T=-\sum_{u=0}^{M-1} \sum_{v=0}^{N-1}|F(u, v)|^{2} \log |F(u, v)|^{2}
$$

When the frequency domain components more concentrated, the greater the frequency domain entropy $T$, that is to say, after dividing the amount of information obtained from the original figure greater segmentation more detailed images, and thus better overall segmentation.

Let threshold $\varepsilon$, if the value more than $\varepsilon$ which is the target area, otherwise is the background area. Spatial frequency energy of pests $E(O)$ and vegetable leaf region $E(B)$ is calculated in this paper, corresponding, frequency domain entropy are $T(O)$ and $T(B)$ respectively. Image entropy function defined as,

$$
T(I)=T(O)+T(B)
$$

The canonical form of the Shannon entropy is defined as follows.

$$
H(p)=-k \sum_{i=1}^{n} p_{i} \log \left(p_{i}\right) \text {, }
$$


where $P=\left\{p_{1}, p_{2}, \cdots, p_{n}\right\}$ is a probability distribution and $k$ is a positive constant. We let $k=1$ and adopt base 2 logarithms. Two different image entropy measures have been suggested in the literature by employing different ways of defining the probability distribution $P$ for images.

Suppose that there are $n$ levels of grayscale in an image $I$. The first order grayscale histogram of $I$ and let $p_{i}$ equals the frequency of the occurrence of grayscale $i(i=1,2, \cdots, n)$.We name entropy thus achieved the 'image histogram entropy' and denote it as $H_{h}(I)$. The image histogram entropy has been used for detecting edges in texture images.

Although both the image histogram entropy and the image grayscale entropy have been widely used as image entropy measures in the literature, they differ significantly in both physical implications and numerical properties.

Image segmentation is the process of dividing an image into multiple parts. This is typically used to identify objects or other relevant information in digital images. There are many different ways to perform image segmentation, including Thresholding methods, Color-based Segmentation, Transform methods, and Texture methods.

\section{On Line Analysis of Image Data}

Agricultural image data contains a crop growth characteristic information, texture information and shape information. Use above complex information, online analysis of image data can mine some spatial characteristic rules, spatial distribution and spatial knowledge for agricultural production.

Online image analysis, firstly, the multiple layers association are obtained by linear regression equation, then, build a regression equation, the association characteristics are mined of the image and crop growing from image data.

Combining analysis by texture, shape, combining semantic feature, and define the agricultural image data of four vectors with original quad data, texture, shape, semantics.

$I=I(D, T, S, L)$

$\omega_{T}, \omega_{S}, \omega_{L}$, are normalization weight of texture, shape, semantic, the above four tuple constituting the image analysis model.

$R=(D, T, S, L, M)$

Where $M=\omega_{T} D_{T}+\omega_{S} D_{S}+\omega_{L} D_{L}, \omega_{T}+\omega_{S}+\omega_{L}=1$

$D_{T}, D_{S}, D_{L}$, are Gaussian normalized distance of texture, shape and semantic feature. $\omega_{T}, \omega_{S}, \omega_{L}$ are assigned weight of texture, shape, semantic.

Take depending on the analysis requirements weighted and dynamic allocation of shape feature, right texture features and semantic features heavy solve combination image feature normalization and weighting dynamically allocated two key issues, the results of the analysis texture and shape characteristics than single use more in line with the human visual perception requirements, improve analytical performance.

Meanwhile, according to user feedback, the establishment of model inspired to explore a heuristic multi-feature fusion, from agriculture image semantic feature analysis, the weight of each feature weight corresponding adjustment heuristic parameter optimization process, whereby a better solution human-computer interaction issues, in favor of an effective system to help users understand the semantics, improve the analysis accuracy.

Traditional relational data mining is a knowledge-user-centered, Human-Computer Interaction process of exploration. Due to the characteristics of agricultural image data, the mining process of image data is special. It is mainly reflected in the need for feature extraction of agricultural production sensing image and setting up feature database. The treatment thought of data warehouse and related technology make sensing image processing can be set up in a more efficient and flexible data mining system. As shown in Figure 1, the main flow briefly describe as follows. 


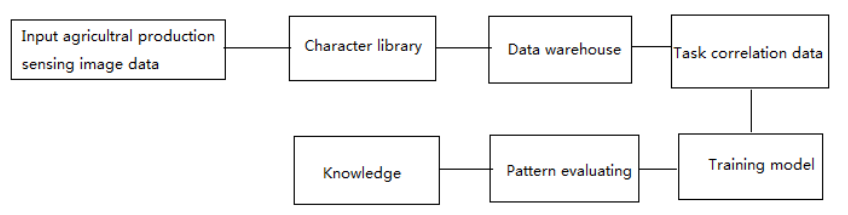

Figure 1. $\quad$ Process of image data analysis

Data analysis model is an abstract data structure, created, filled and provided query by data analysis algorithm. Through the input and output strings of specified data analysis model and the use of analysis algorithm, data analysis algorithm can set up an empty data analysis model structure; and through inserting the training set into data analysis model, the training of the model is implemented.

\section{Simulation Results and Analysis}

In order to verify the performance of algorithm FSC, we select large scale images and images from Berkeley image database, where large scale images are obtained by the author. Their size is $1024 \times 768$. The size of images from Berkeley image database is $481 \times 321$. The experimental environment is CPU2.0G, memory 1.0G and Matlab7.1.

Effectively dividing the adjacent divided regions should have high contrast; select the contrast between the gray areas. Comparison the optimal threshold value and the gray area, the result as showed in table 1.

Table 1. Comparatice of optimal threshold and gray contrast across the region by different image

\begin{tabular}{|l|l|l|l|l|}
\hline image & Leaf1.jpg & Fruit1.jpg & Leaf2.jpg & Fruit1.jpg \\
\hline threshold & 37 & 72 & 128 & 74 \\
\hline GC & 0.8422 & 0.5659 & 0.3919 & 0.7553 \\
\hline
\end{tabular}

Contrast between the gray areas is defined as $G C=\left|f_{O}-f_{B}\right| /\left(f_{O}+f_{B}\right)$, where $f_{O}$ and $f_{B}$ is mean gradation of the target object and background respectively. And GC is larger value, the segmentation is better. From time performance analysis, time of getting the optimal threshold is reduced in this paper, and time complexity is $O\left(L^{3}\right)$, and CPU time is not correlated with the size of the image. Regardless of image size, CPU time consuming is generally between $0.4-0.5$ s, as showed in table 2.

Table 2. Performance comparison of cpu time for different image

\begin{tabular}{|l|l|l|l|l|}
\hline image & $\begin{array}{l}\text { Leaf1.jpg } \\
(\mathbf{1 0 2 4} * \mathbf{1 0 2 4})\end{array}$ & $\begin{array}{l}\text { Leaf1.jpg } \\
\mathbf{( 1 0 2 4 * 1 0 2 4 )}\end{array}$ & $\begin{array}{l}\text { Leaf2.jpg } \\
\mathbf{( 5 1 2} * 512)\end{array}$ & $\begin{array}{l}\text { Fruit1.jpg } \\
\mathbf{( 5 1 2 * 5 1 2 )}\end{array}$ \\
\hline CPU time & 0.5495 & 0.5755 & 0.5313 & 0.5451 \\
\hline
\end{tabular}

\section{Conclusions}

The application of image processing has widely applied in our life, in which the digital image processing technology is widely used in all aspects of life. Image segmentation is a key step in transition to the image analysis as low level processing in digital image processing. In this paper we have proposed a fast image segmentation algorithm based on spectral clustering which can process large size image. In the experiment, we verify the effectiveness of the presented method and compare it other methods. Subjective and objective analysis shows that this algorithm can effectively distinguish different textures, while the direction of edge information has been well retained. Visual effect and evaluation parameters have already illustrated the effectiveness of the algorithm. Getting precise segmentation results from the image will provide an effective basis for future target recognition and testing.

\section{Acknowledgement}

This work was supported by Beijing Municipal Natural Science Foundation (4151001). 


\section{References}

[1] G. Eason, B. Noble, and I. N. Sneddon, "On certain integrals of Lipschitz-Hankel type involving products of Bessel functions,” Phil. Trans. Roy. Soc. London, vol. A247, pp. 529-551, April 1955.

[2] J. Clerk Maxwell. “A Treatise on Electricity and Magnetism, ” 1992,2(3), pp.68-73

[3] I. S. Jacobs, C. P. Bean. "Fine particles, thin films and exchange anisotropy,” Magnetism, vol. III, G. T. Rado and H. Suhl, Eds. New York: Academic, 1963, pp.271-350.

[4] Y. Yorozu, M. Hirano, K. Oka, et al. "Electron spectroscopy studies on magneto-optical media and plastic substrate interface, ” IEEE Transl. J. Magn, 1987,2, pp.740-741

[5] Lina Yi, Guifeng Zhang, et al. "A Scale-Synthesis Method for High Spatial Resolution Re-mote Sensing Image Segmentation,"IEEE Transactions on Geoscience and Remote Sensing, 2013,50(10), pp.4062- 4070

[6] Luming Zhang, Yue Gao, et al. "Representative Discovery of Structure Cues for Weakly-Supervised Image Segmentation”. IEEE Transactions on Multimedia, 2014,16(2), pp.470-479

[7] Lichao Wang, Lekadir, K, et al. “A General Framework for Context-Specific Image Segmenta-tion Using Reinforcement Learning, ” IEEE Transactions on Medical Imaging, 2013, 32(5) , pp. 943 - 956

[8] Koosha, M., Hajsadeghi, K., et al. "Fine logarithmic adaptive soft morphological algorithm for synthetic aperture radar image segmentation,” IET Image Processing, 2014,8(2) , pp. 90-102

[9] Yu Sun, Bhanu, B. "Reflection Symmetry-Integrated Image Segmentation. IEEE Transactions on Pattern Analysis and Machine Intelligence, ” 2012,34(9) , pp.1827- 1841

[10]Bova, N.,Ibanez, O., et al. "Image Segmentation Using Extended Topological Active Nets Optimized by Scatter Search,” IEEE Computational Intelligence Magazine,2013,8(1) , pp. 16-32

[11]Despotovic, I.,Vansteenkiste, E., et al. "Spatially Coherent Fuzzy Clustering for Accurate and Noise-Robust Image Segmentation,” IEEE Signal Processing Letters, 2013,20(4) , pp. 295- 298

[12]Khokher, M.R.,Ghafoor, A.,Siddiqui, A.M. "Image segmentation using multilevel graph cuts and graph development using fuzzy rule-based system,” IET Image Processing, 2013,(7)3, pp.201211 